\title{
Jahresbericht über die Aktivitäten der FMH im Bereich MPA
}

\author{
Adrian Sury \\ Dr. med., Präsident der kantonalen Delegierten für MPA-Fragen
}

In diesem Jahr hat es in der Aufgabenkommission QV MPA einen personellen Wechsel gegeben. Dr. Katrin Hubschmid hat sich nach mehrjährigem intensivem Engagement als Fachgruppenleiterin ATMB und als Präsidentin der Aufgabenkommission QV MPA zurückgezogen. Für ihren grossen, unermüdlichen Einsatz sei ihr an dieser Stelle noch einmal ganz herzlich gedankt. Im Januar 2017 konnte Dr. Gert Printzen, Fachgruppenleiter ATMB in der Aufgabenkommission QV MPA, für das Präsidium gewonnen werden. Ich wünsche ihm viel Erfolg in dieser anspruchsvollen Aufgabe.

Die Aufgabenkommission Qualifikationsverfahren Medizinische Praxisassistentin (QV MPA) erarbeitet unter grossem Einsatz die Prüfungsunterlagen. Das Erarbeiten einer gesamtschweizerischen einheitlichen Prüfung stellt die Fachgruppen und insbesondere die Fachgruppenleiterinnen und Fachgruppenleiter immer wieder vor eine grosse Herausforderung, gibt es doch in einzelnen Fachgebieten nicht zu unterschätzende Unterschiede der Gepflogenheiten zwischen der Romandie, dem Tessin und der Deutschschweiz.

Die Anliegen der verschiedenen Gremien an den Zentralvorstand der FMH werden weiterhin durch Dr. Carlos Quinto, Mitglied Zentralvorstand der FMH und Departementsverantwortlicher Public Health und Gesundheitsberufe, vertreten.

Aktuell sieht die Aufgabenteilung neu wie folgt aus:

- Delegierte der kantonalen Ärztegesellschaften für MPA-Fragen: Präsident Dr. A. Sury, Vizepräsident Dr. R. Tognina

- Schweizerische Kommission für Berufsentwicklung und Qualität (Kommission B \& Q): Präsident Dr. J. Orellano

- Aufgabenkommission für das Qualifikationsverfahren (QV) MPA: Präsident Dr. G. Printzen

- OdA Berufsbildung MPA (odamed): Präsidentin Frau M. Schenk/SVA, Dr. G. Printzen, Vizepräsident

Das MPA-Büro, das aus den oben aufgeführten Gremienverantwortlichen der FMH besteht, trifft sich in der Regel einmal jährlich oder ad hoc, um allfällige Probleme, Überschneidungen oder Projekte zu besprechen.
Wir können auf ein ereignisreiches Jahr zurückblicken, wie die nachfolgenden Berichte der Verantwortlichen in den verschiedenen Kommissionen zeigen.

\section{Schweizerische Kommission für Berufsentwicklung und Qualität (Dr. José Orellano)}

In diesem Jahr wurde die neue Bildungsverordnung MPA weiter forciert. Eine Vernehmlassung bei den kantonalen Ärztegesellschaften, der Schweizerischen Berufsbildungsämter-Konferenz (SBBK), den Ausbildungsstätten sowie den MPA-Verbänden (SVA, ARAM) hat diese Bildungsverordnungsvorlage weiter zur Reifung gebracht. Die Eingaben für die Totalrevision der Bildungsverordnung wurden bereits gemacht. Die Inkraftsetzung der Bildungsverordnung ist für den 1.1.2019 geplant. Das Staatssekretariat für Bildung, Forschung und Innovation (SBFI) führt derzeit bei den für die Berufsbildung zuständigen kantonalen Ämtern, den Organisationen der Arbeitswelt und weiteren interessierten Kreisen eine externe Vernehmlassung durch. Anschliessend werden die letzten Anpassungen für die neue Bildungsverordnung implementiert und zur definitiven Genehmigung eingegeben, so dass wir wie geplant 2019 mit der neuen Ausbildung beginnen können. Ebenfalls ist der zugehörige Bildungsplan praktisch vollständig überarbeitet und neu in fünf Handlungskompetenzbereiche eingeteilt, welche die Arbeit der MPA konkret abbilden. Es wurde versucht, die praktischen Arbeiten im QV höher zu gewichten. Wir hoffen, dass diese Massnahme das Niveau des EFZ entsprechend aufwertet.

Der sogenannte Anhang 2 der Bildungsverordnung «Begleitende Massnahmen der Arbeitssicherheit und des Gesundheitsschutzes» wurde am 1.4.2017 in Kraft gesetzt. Zum bisherigen Anhang wurde neu der Schutz für Jugendliche besonders beachtet. Neu dürfen unter 16-jährige Auszubildende sich nicht während der Exposition in Röntgenräumen aufhalten. Auch mögliche Exposition mit Reizstoffen und/oder Handhabung von Zytostatika sind bei unter 16-Jährigen zu vermeiden. Die in der Verordnung enthaltenen Massnahmen können im Anhang 2 weiter studiert werden. Kantonale In- 
formationsveranstaltungen durch die entsprechenden Ärztegesellschaften in Zusammenarbeit mit Schulen und Lehrlingsämtern sind zu begrüssen. Es wird empfohlen, dass Berufsbildnerinnen und Berufsbildner diese Veranstaltungen besuchen, damit die Ausbildung für die Jugendlichen sicherer wird.

\section{Weiterbildung MPA - Bericht aus der OdA Berufsbildung MPA (Dr. Gert Printzen)}

Die Weiterbildung Medizinische Praxiskoordinatorin MPK ist mittlerweile etabliert und erfreut sich eines zunehmenden Interesses. Wiederum hat die Anzahl der Prüfungsanmeldungen zur MPK-Weiterbildung die Erwartungen übertroffen. Wie auch in der vergangenen Berichtsperiode wurden diese obligaten Prüfungen für MPK im November und im Juni durchgeführt. Am 24. November 2016 hat die dritte Berufsprüfung zur Medizinischen Praxiskoordinatorin mit 8 Kandidatinnen klinischer und 38 Kandidatinnen praxisleitender Fachrichtung stattgefunden. 40 dieser 46 Kandidatinnen haben die Prüfung bestanden.

Am 8. Juni 2017 traten zur 4. Berufsprüfung zur Medizinischen Praxiskoordinatorin 17 Kandidatinnen klinischer und 25 Kandidatinnen praxisleitender Fachrichtung an. 35 der 42 Kandidatinnen haben die Prüfung bestanden und konnten den eidgenössischen Fachausweis MPK entgegennehmen; hierunter befanden sich 6 Repetentinnen, von denen 5 ebenfalls erfolgreich waren. Im Rahmen der zugehörigen Prüfungsfeier gratulierte die odamed-Präsidentin Marianne Schenk den Absolventinnen zum Erfolg nach einem anstrengenden Studium mit dem Besuch von sechs Modulen und rund 300 Lektionen. Sie machte zudem auf die Herausforderungen der Digitalisierung in der Arztpraxis aufmerksam und mahnte im Interesse der Wahrung einer hohen Arbeitsqualität zur Entschleunigung der Arbeitsprozesse.

Zum kommenden Prüfungstermin im November 2017 sind bereits erfreuliche 93 Personen (32 klinisch, 62 praxisleitend) angemeldet; das erste Mal werden auch Teilnehmerinnen aus der Romandie antreten.

Im Jahre 2018 sind die Prüfungstermine erneut für Juni und November festgelegt.

In der Westschweiz wurden für die MPK-Weiterbildung erste Anbieter mit einem Modul akkreditiert.

- Einerseits ist dies die ARAM (Association Romande des Assissantes Médicales) in Lausanne mit der Anerkennung durch das BAG (Bundesamt für Gesundheit) für das Modul Dosisintensives Röntgen und

- anderseits die Espace Compétences SA in Cully mit der Anerkennung für die Module Chronic Care
Management I + II, Qualitätsmanagement in der Arztpraxis, Wundbehandlung, Wiederaufbereitung von Medizinprodukten, Beratung von Langzeitpatienten (Diabetes, Rheuma, Koronare Herzerkrankungen/Herzinsuffizienz, Hirnleistungsschwäche), Praxismanagement, Rechnungswesen, Personalführung.

Im Tessin gibt es Entsprechendes seit Sommer 2016 bei der Scuola Superiore Medico-Tecnica SSMT in Locarno, mit Anerkennung ausgesprochen für die Module Wiederaufbereitung von Medizinprodukten, Chronic Care Management I und Anerkennung durch das Bundesamt für Gesundheit BAG für das Modul Dosisintensives Röntgen.

$\mathrm{Zu}$ den Finanzen der odamed lässt sich konstatieren, dass diese praktisch ausgeglichen sind. Die eigentliche Prüfung ist zusammen mit den Prüfungsgebühren und den Bundesbeiträgen selbsttragend.

Die MPK-Betriebsphase präsentiert sich erfolgreich. Die Perfektionierung wird weiter gesteigert - dazu gehören die Optimierung der Website www.odamed.ch und weiter der Ausbau auf drei Sprachen, die Beendigung aller Übersetzungsarbeiten und die Akkreditierung weiterer Bildungsanbieter und Module. Weiterhin werden Expertinnen und Experten in allen drei Landessprachen gesucht, die sich bei der Geschäftsstelle melden sollen. Diese Expertinnen und Experten werden adäquat für ihre spezifische Aufgabe geschult.

\section{Aufgabenkommission für das Quali- fikationsverfahren (QV) MPA (Dr. Gert Printzen)}

Das Qualifikationsverfahren 2017 konnte auch in diesem Jahr ohne schwerwiegende Probleme durchgeführt werden.

Auch dieses Jahr zeigt der leichte Anstieg der Ungenügenden, dass die Prüfung nicht allzu einfach war, was ja in den vergangenen drei Jahren so bezweckt wurde. Nach wie vor existiert das Problem, dass die Ergebnisse des Qualifikationsverfahrens im Schlusszeugnis, dem Eidgenössischen Fähigkeitszeugnis EFZ, nicht detailliert aufgeführt sind. Somit sieht man nicht, wenn eine ungenügende Note in einem der Hauptfächer durch einen genügenden Durchschnitt verschleiert respektive überdeckt wird. Hierzu gibt es immer noch diverse Beanstandungen, vor allem bezüglich der «Bildgebenden Diagnostik».

In der Labordiagnostik und der bildgebenden Diagnostik verändern sich die Anforderungen unter anderem 
durch automatisierte Analysegeräte, aber auch z.B. durch digitales Röntgen zunehmend. Einerseits wird zu klären sein, welche Fähigkeiten für das Qualifikationsverfahren obligat sind, und daraus sind die Konsequenzen zu ziehen, welche Fähigkeiten aufgegeben werden müssen oder können. Entsprechend sind dann Ausbildung und Prüfungen anzupassen.

Vergleichbares gilt auch für die «Betrieblichen Prozesse»: Die Digitalisierung ist nicht in allen Praxen und Betrieben gleich etabliert. Daraus resultieren grosse Unterschiede und somit auch verschiedene Ansprüche an die Qualifikation.

Der Aufwand für die Prüfungen war wie in den Vorjahren auch 2017 beträchtlich und nur dank des grossen Einsatzes aller Beteiligten zu bewältigen. Ich danke allen Mitwirkenden für ihr ausserordentliches Engagement ganz herzlich.

\section{MPA-Ausbildung}

Im Jahr 2017 wurden 1028 MPA-Kandidatinnen geprüft. Von diesen 1028 Kandidatinnen haben 950 (92,6\%) die Prüfung erfolgreich bestanden. kanntermassen nicht auf Grundversorgerpraxen mit Labor und Röntgen beschränkt, sondern steht ebenso anderen Fachbereichen offen. Dies gilt auch für Spitäler und Kliniken, die im Rahmen der ambulanten Leistungen Ausbildungsstellen anbieten - positive Rückmeldungen hierzu gibt es aus dem Kanton Tessin und nun auch aus Zürich. Hingewiesen werden soll auch nochmals auf die Möglichkeit, im Rahmen von Kooperationen mit einer Klinik, einem Spital oder im Verbund mit anderen Praxen Teilbereiche des Berufes, z.B. bildgebende Diagnostik oder Labordiagnostik, ergänzend zu erlernen. Auskünfte hierzu erteilt das kantonale Amt für Berufsbildung.

Für MPA, die sich als Berufsbildnerinnen weiterbilden möchten, organisiert der Schweizerische Verband Medizinischer Praxis-Fachpersonen SVA entsprechende Kurse. In einigen Kantonen werden solche Berufsbildnerkurse auch von den Kantonen selbst angeboten.

\section{Aktuell / Hinweise}

\section{Dosisintensives Röntgen}

Ich möchte die Ausbildung für dosisintensive Röntgen-

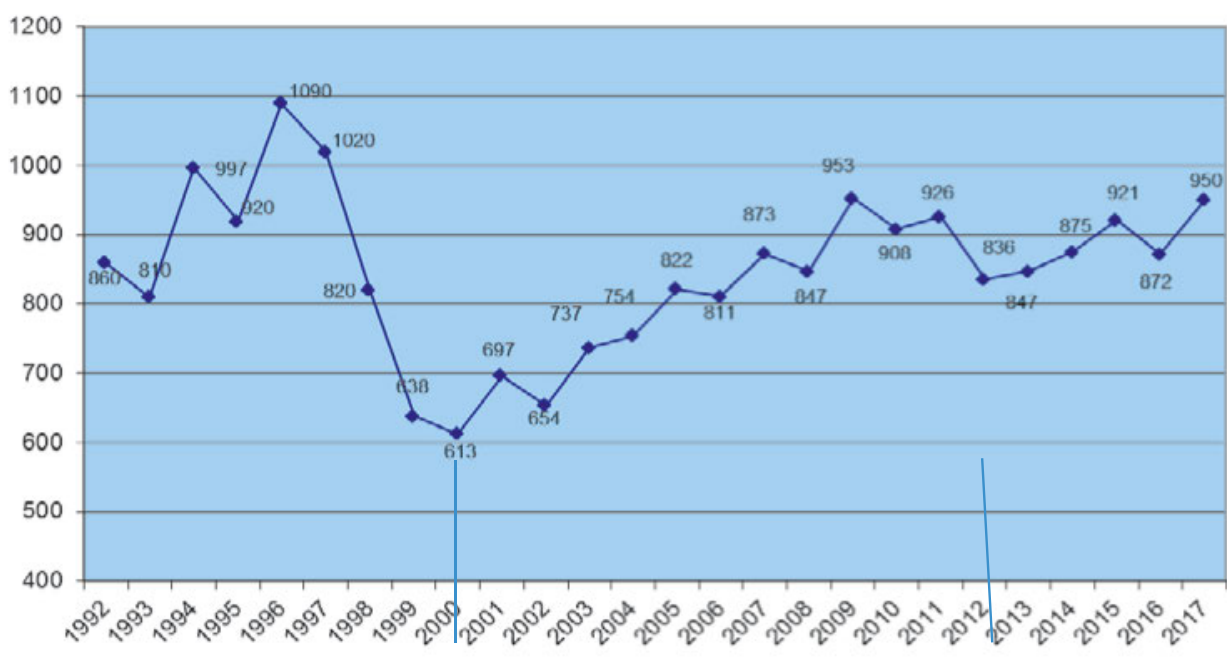

Das Interesse und die Nachfrage nach Lehrstellen haben sich - wahrscheinlich mit der Perspektive der Weiterbildung zur MPK (Medizinische Praxiskoordinatorin) - verstärkt. Um auch zukünftig genügend Berufsleute ausbilden zu können, müssen weiterhin entsprechende Lehrstellen zur Verfügung stehen. Die Möglichkeit, eine Ausbildungsbewilligung durch das kantonale Amt für Berufsbildung zu erhalten, ist be- untersuchungen in Erinnerung rufen. Die Erfüllung der Ausbildungspflicht für MPA und Ärzte wird vom BAG kantonal und regional überprüft. Falls dies nicht bereits geschehen ist, bitten wir Sie als Ärzte und Ihre MPA, einen entsprechenden Kurs zu besuchen, um sowohl Unannehmlichkeiten mit dem BAG zu vermeiden als auch mitzuhelfen, die Patientensicherheit zu gewährleisten. 
SwissSkills (12.-16. September 2018, Bern)

Im Jahre 2014 wurden erstmals zentrale Schweizer Berufsmeisterschaften, die SwissSkills, durchgeführt. Diese erfolgreiche nationale Grossveranstaltung für das Schweizerische Berufsbildungssystem fand grossen Anklang bei allen Beteiligten und in der Öffentlichkeit.

Die kommenden SwissSkills 2018 finden vom 12.16. September 2018 in den Messehallen der BERNEXPO in Bern statt. Mit einem gemeinsamen Informationsstand wollen die FMH, der SVA, unter Beteiligung weiterer Organisationen wie mfe und JHAS von ärztlicher Seite und ARAM von MPA-Seite, einen Einblick in den MPA-Beruf geben. Am Stand sollen Ärztinnen, Ärzte sowie Vertreter von ARAM und SVA anwesend sein.

Mit der Präsenz an den SwissSkills möchten wir dazu beitragen, Werbung für den MPA-Beruf zu machen. Junge Leute sollen motiviert werden, diesen interessanten Beruf zu erlernen, was wiederum dazu führen soll, dem schweizweiten MPA-Mangel entgegenzuwirken.

\section{www.mpaschweiz.ch - www.fmh.ch}

Die Website www.mpaschweiz.ch enthält ausschliesslich Informationen und nützliche Dokumente zum Download im Zusammenhang mit der Ausbildung von MPA. Die laufend aktualisierte Seite wird vom MPA-Sekretariat der FMH betreut. Unter www.am-suisse.ch kann auf die französische Version zugegriffen werden. Unter www.fmh.ch $\rightarrow$ Services $\rightarrow$ Medizinische Praxisassistentin sind die Mustervorlagen und Hinweise im Rahmen der Anstellung von diplomierten MPA aufgeschaltet (Musterarbeitsvertrag, Lohnempfehlungen, Mutterschutz).

Korrespondenz:

Dr. med. Adrian Sury Präsident der kantonalen Delegierten für MPA-Fragen Elfenstrasse 18 CH-3000 Bern 15 die Deutschschweiz, Rechtsanwalt und Notar lic. iur. A. Kummer, eingegangen, noch sind Fälle hängig.

\section{MPA-Sekretariat im Generalsekretariat}

Der Informationsfluss zwischen den verschiedenen Akteuren und ihren Tätigkeitsgebieten sowie dem Zentralvorstand ist durch Elisabeth Tröhler vom MPASekretariat der FMH gewährleistet.

Sie steht sowohl dem Präsidenten der MPA-Delegierten als auch Dr. Carlos Quinto für alle Geschäfte im Bereich MPA zur Verfügung. Daneben führt sie insbesondere die Sekretariate der Aufgabenkommission QV MPA, der Kommission B \& Q und der Aufsichtskommission ÜK. Das MPA-Sekretariat ist eng mit der Abteilung Rechtsdienst der FMH verknüpft, womit auch die Überarbeitung von Reglementen und Vereinbarungen gewährleistet ist.

\section{Zum Schluss}

Auch dieses Jahr darf ich all jenen danken, die sich tatkräftig für die Belange der MPA eingesetzt haben. Mein Dank richtet sich aber auch an alle ausbildenden MPA und BerufsbildnerInnen, die Schulen und die MPA-Verbände. Ganz speziell herzlich möchte ich auch Frau Elisabeth Tröhler vom MPA-Sekretariat für ihr grosses Engagement und ihre Kompetenz danken.

Im Berichtsjahr hat sich auch die Ausbildung zu den MPK konsolidiert. In den Kantonen scheinen endlich auch die MPA ihre Anerkennung auf Gesetzesebene zu finden. Wir hoffen nun, dass dies zu einer Abbildung im Tarifwesen führen wird, damit die gesteigerte Kompetenz auch angemessen entlohnt werden kann.

Wir Ärzte und Spitäler müssen aber genügend Lehrstellen offerieren, um der Nachfrage zu genügen. Die Anstrengungen der FMH müssen sich in der nächsten Zukunft vor allem auf diese Problematik fokussieren und möglicherweise auch neue Finanzierungsmodelle der Ausbildung evaluieren! 